\title{
Effect of Sevelamer and Nicotinamide on Albumin Carbamylation in Patients with End-Stage Kidney Disease
}

\author{
Aurelie Lenglet $^{1,2} \cdot$ Mohamad Ali Rahali $^{3} \cdot$ François-Ludovic Sauvage $^{3} \cdot$ Sophie Liabeuf ${ }^{1,4} \cdot$ Gabriel Choukroun $^{1,5}$. \\ Marie Essig ${ }^{6,7}$. Souleiman El Balkhi ${ }^{3,8}$. Ziad A. Massy ${ }^{6,7}$
}

Accepted: 17 May 2021 / Published online: 8 June 2021

(c) The Author(s) 2021, corrected publication 2021

\begin{abstract}
Background and Objective In end-stage kidney disease, high urea levels promote the carbamylation of lysine side chains on a variety of proteins, including albumin. Albumin carbamylation has been identified as a risk factor for mortality and sevelamer led to a decrease in urea levels in dialysis patients. In the present secondary analysis of the NICOREN trial, we investigated the putative impacts of sevelamer and nicotinamide on albumin carbamylation, and the potential correlation between carbamylation and vascular calcifications.

Methods All possible carbamylation of circulating albumin were screened for with high-resolution liquid chromatographytandem mass spectrometry. Levels of three carbamylated peptides were then measured as a guide to the extent of albumin carbamylation. Carbamylation was measured at baseline in 55 patients included in the NICOREN trial and 29 patients at 24 weeks of treatment. Calcifications on plain radiographs were quantified as the Kauppila score and the Adragao score.

Results Baseline albumin carbamylation was present at three different sites in subjects with end-stage kidney disease. At baseline, we observed only a correlation between urea and the KQTA carbamylation site in these patients. Albumin carbamylation levels did not decrease after 24 weeks of treatment with either sevelamer or nicotinamide. Furthermore, the proportion of carbamylated serum albumin was not correlated with vascular calcification scores in this population.

Conclusions Our results confirmed the presence of carbamylated albumin in patients with end-stage kidney disease and demonstrated the presence of carbamylation beyond the LRVP residues. The results also demonstrated the lack of impact of sevelamer or nicotinamide on albumin carbamylation levels. Therapeutic strategies to lower carbamylation load should probably be focused on direct anti-carbamylation processes and/or potentially anti-inflammatory therapies.
\end{abstract}

\section{Key Points}

Carbamylation has detrimental effects at all physiological levels, and its particular relationship with inflammation and uremia sheds light on mortality risk factors in patients with end-stage kidney disease.

Carbamylated albumin is present in patients with endstage kidney disease, and residues outside albumin's LRVP sequence are carbamylated.

The administration of sevelamer or nicotinamide does not influence carbamylation levels.

Aurelie Lenglet and Mohamad Ali Rahali are joint first authors.

Ziad A. Massy

ziad.massy@aphp.fr

Extended author information available on the last page of the article 


\section{Introduction}

Patients with end-stage kidney disease (ESKD) suffer disproportionately from cardiovascular disease. Conventional risk factors (such as hypertension, atherosclerosis, and hypercholesterolemia) are highly prevalent in patients with ESKD and contribute to the cardiovascular risk. However, newly identified, non-conventional risk factors (such as protein carbamylation) might also be mechanistically linked to cardiovascular disease [1]. Several studies have suggested that carbamylation and/or carbamoylation can contribute to atherosclerosis and the cardiovascular disease risk via its effects on lipoproteins, collagen, fibrin, proteoglycans, and fibronectin $[1,2]$. In the literature, the terms "carbamoylation" and "carbamylation" are sometimes used erroneously as synonyms. In fact, carbamylation refers to a different chemical reaction: the reversible interaction of $\mathrm{CO}_{2}$ with $\alpha$-amino and $\varepsilon$-amino groups in proteins. Protein carbamylation is a non-enzymatic posttranslational protein modification that can be driven (at least in part) by exposure to cyanate (a product of urea dissociation). Carbamylated compounds are formed by the reaction of proteins, peptides, or amino acids with isocyanic acid derived from urea or by oxidation of thiocyanate by myeloperoxidase in an inflammatory setting and in atherosclerotic plaques [2]. When kidney function is impaired and urea accumulates in the body, the level of systemic protein carbamylation increases [3]. The type of carbamylation product depends on the altered molecule (i.e., albumin, collagen, erythropoietin, hemoglobin, lowdensity lipoprotein, and high-density lipoprotein), and thus carbamylation can have various harmful effects [2]. The carbamylation of protein side-chain lysines is promoted by urea. The predominant carbamylation site on albumin is lysine 549. In a recent animal study, it was shown that protein carbamylation exacerbates medial vascular calcification by downregulating the expression of ectonucleotide pyrophosphate/phosphodiesterase 1 (ENPP1), a key enzyme in the generation of pyrophosphate, a potent inhibitor of ectopic calcification [4]. Moreover, the serum level of carbamylated albumin is specifically associated with excess mortality in patients with ESKD and diabetes mellitus [5, 6].

Via the NICOREN trial in a cohort of patients with ESKD, we have previously assessed the efficacy and safety of the phosphate binder sevelamer vs the absorption inhibitor nicotinamide [7]. We found that 24 weeks of treatment with sevelamer led to a significant decrease in the blood urea level [8]. Sevelamer has been shown to exert a number of potentially beneficial pleiotropic actions, some of which may improve cardiovascular functions [9]. The drug reduces the absorption of advanced glycation end-products, bacterial toxins, and bile acids; hence, it may reduce inflammatory, oxidative, and atherogenic stimuli in addition to its direct phosphate-lowering action. To the best of our knowledge, however, the effects of sevelamer on protein carbamylation have not previously been investigated. The vitamin $B_{3}$ derivative nicotinamide is a potentially interesting pharmacological alternative to binder-based approaches in the treatment of hyperphosphatemia [10]. It is involved in a wide range of biological processes, including energy production, the synthesis of fatty acids, cholesterol, and steroids, signal transduction, and the maintenance of genomic integrity. Furthermore, nicotinamide reportedly has anti-inflammatory properties [11-14].

The objectives of the present secondary analysis of the NICOREN trial were to (i) determine whether treatment with sevelamer or nicotinamide was associated with the degree of serum albumin carbamylation (as quantified by liquid chromatography-tandem mass spectrometry) and (ii) assess a putative link between vascular calcification and albumin carbamylation in patients with ESKD.

\section{Materials and Methods}

\subsection{Patient Selection}

This was the secondary analysis of the NICOREN trial data [7]. The trial included 100 patients undergoing long-term hemodialysis treated with either sevelamer- $\mathrm{HCl}$ or nicotinamide for 24 weeks. The patients were dialyzed three times per week for 3.5-5.0 h. The study was performed in 18 hemodialysis centers across France. All patients gave their informed written consent to the initial and subsequent biochemical analyses. The protocol was approved by a local investigational review board (CPP Nord Ouest II, Amiens, France; reference: 2008-004673-17) and implemented in accordance with the ethical principles of the Declaration of Helsinki (ClinicalTrials.gov registration number: NCT01011699).

\subsection{Laboratory Tests}

Serum urea, albumin, and protein were assayed in an onsite biochemistry laboratory using standard auto-analyzer techniques. After enrollment, serum was obtained and immediately frozen at $-80^{\circ} \mathrm{C}$ prior to analysis (i.e., carbamylated protein measurements) at the end of the study in the Biobanque de Picardie Biological Resource Center. In this study, serum samples were available for 55 patients at baseline (27 patients in the sevelamer group and 28 patients in the nicotinamide group) and 29 patients at 24 weeks of treatment (19 patients in the sevelamer group 
and ten patients in the nicotinamide group). For the 45 patients not included in the analysis (because the sample was not available), there was no significant difference in age $(63.2 \pm 14.5$ years vs $66.4 \pm 13.1$ years; $p=0.247)$, serum urea $(23.6 \pm 6.4$ vs $25.5 \pm 8.1 ; p=0.197)$, serum albumin (37.8 \pm 9.9 vs $37.5 \pm 3.4 ; p=0.918)$, or dialysis dose [Kt/Vurea] (1.29 \pm 0.32 vs $1.28 \pm 0.24 ; p=0.918)$.

\subsection{Preparation of Serum Samples}

For each serum sample, a volume containing $100 \mu \mathrm{g}$ of protein was treated with $100 \mu \mathrm{L}$ of dithiothreitol $(100 \mathrm{mM}$ in $8 \mathrm{M}$ of urea solution, $\mathrm{pH} 8.0)$ and incubated at $56^{\circ} \mathrm{C}$ for $30 \mathrm{~min}$. Next, $100 \mu \mathrm{L}$ of iodoacetamide $(100 \mathrm{mM}$ in 8 $\mathrm{M}$ of urea solution, $\mathrm{pH}$ 8.0) were added, and the sample was incubated for $30 \mathrm{~min}$ in the dark at room temperature. One microgram of trypsin (mixed in $50 \mathrm{mM}$ of ammonium bicarbonate solution) was added, and the sample was incubated again at $37{ }^{\circ} \mathrm{C}$ for $3 \mathrm{~h}$. Last, the digested peptides were eluted by centrifugation after the addition of $3 \times 40 \mu \mathrm{L}$ of $50 \mathrm{mM}$ of ammonium bicarbonate solution. The tryptic peptides were extracted by solid-phase extraction and stored at $-80^{\circ} \mathrm{C}$ until analysis. Details of the specimen processing can be found in the Electronic Supplementary Material (ESM).

\subsection{Identification and Measurement of Carbamylation}

All possible carbamylations of albumin were searched for using a quadrupole time-of-flight mass spectrometer (TripleTOF ${ }^{\circledR} 5600+$; Sciex, Concord, ON, Canada). The tandem mass spectrometry fragmentation spectra were matched against the Swissprot ${ }^{\mathrm{TM}}$ library using the Paragon ${ }^{\circledR}$ and Mascot ${ }^{\circledR}$ search algorithms provided with ProteinPilot ${ }^{\mathrm{TM}}$ software (Version 5.0; Sciex). Digested tryptic peptides were then separated by liquid chromatography (C18 reversedphase column), and analyzed for carbamylation modifications with mass spectrometry (an 8060 triple-quadrupole mass spectrometer; Shimadzu Corporation, Marne-la-Vallée, France).

The carbamylated and non-carbamylated peptides of interest were then measured by multiple reaction monitoring using tandem mass spectrometry transitions (settings are shown in Table S1 of the ESM) and quantified according to the area under the curve (AUC) (Fig. 1). The percent carbamylation was calculated as follows: percent carbamylation $=($ AUC for the carbamylated peptide/AUC for the non-carbamylated peptide). The percent carbamylation in subjects with normal kidney function using carbamylation sites was reported by Berg et al. at $0.4 \%$ [15]. Details of specimen processing and additional methods for identification and analysis are given in the ESM. In this study, the albumin carbamylation was evaluated
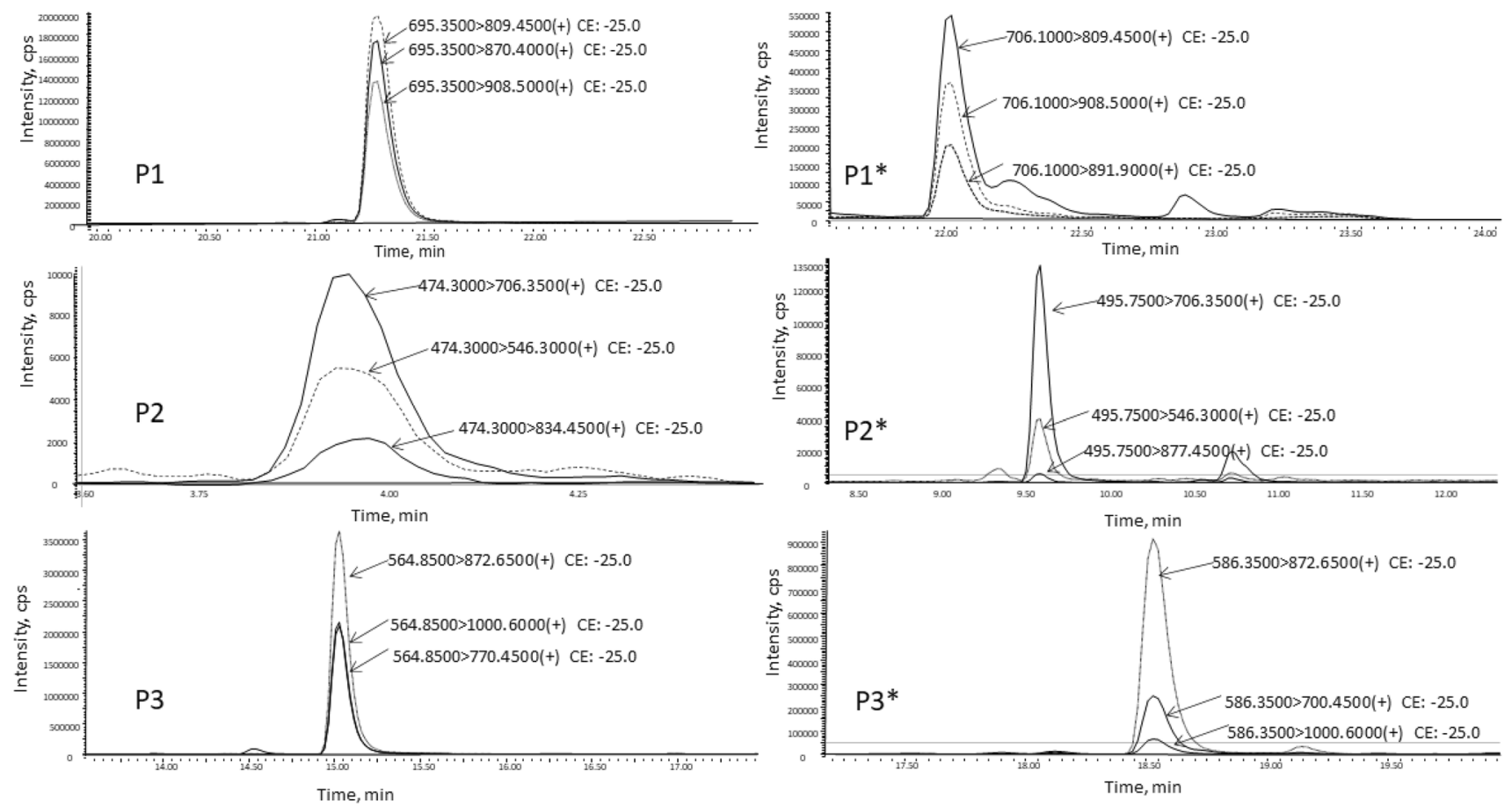

Fig. 1 Mass chromatograms obtained for the three peptides LVRPEVDVMCTAFHDNEETFLKK (P1), LKCASLQK (P2), and KQTALVELVK (P3), and their respective carbamylated peptides $\left(\mathrm{P} 1^{*}, \mathrm{P} 2^{*}\right.$, and $\left.\mathrm{P} 3^{*}\right)$ after the analysis of a serum sample 
in patients with ESKD at baseline and 24 weeks after treatment with sevelamer or nicotinamide.

\subsection{Quantification of Calcification}

Two methods were used to quantify the presence and extent of calcification at baseline: the Kauppila score [16] and the Adragao score [17]. The Kauppila score was applied to images of the lower abdominal aorta. A lateral plain radiograph of the abdomen was obtained, and the aorta was identified as the tubular structure coursing in front of the anterior surface of the spine. We used a semi-quantitative scoring system; only the abdominal aorta segments in front of the four lumbar vertebras were considered. Points were assigned on a $0-3$ scale (0: absent; 1 : small; 2: moderate; 3 : large), according to the length of the lumbar vertebrae under consideration. Hence, the Kauppila score could vary from a minimum of 0 points to a maximum of 24 points.

The Adragao score quantifies vascular calcification in the iliac and femoral arteries (on a plain X-ray of the pelvis) and in the radial and digital arteries (on a plain X-ray of the hand). The presence of linear calcification in a given section was counted as 1 and its absence was counted as 0 . The final score was the sum of all the subscores and thus ranged from 0 to 8 . All the X-rays were read by two investigators, and a consensus was reached in all cases.

\subsection{Statistical Analyses}

Results were expressed as the mean \pm standard deviation, median (interquartile range), or frequency (percentage), as appropriate. The normality of the distribution of quantitative variables was checked with the Shapiro-Wilk test. The study population was divided into two treatment groups. Potential intergroup differences in mean values were evaluated in an analysis of variance. Comparisons of more than two mean values were assessed in a two-way analysis of variance with repeated measures. If a significant difference was found, Tukey's post-hoc test for multiple comparisons was used to confirm the result. Spearman correlation coefficient was calculated as a guide to the strength of associations after the exclusion of few outliers. The threshold for statistical significance was set to $p<0.05$. All statistical analyses were performed using SPSS software (Version 18.0; SPSS Inc., Chicago, IL, USA) for Windows (Microsoft Corp, Redmond, WA, USA) or SAS ${ }^{\circledR}$ software (Version 9.2, SAS Institute Inc., Cary, NC, USA).

\section{Results}

Fifty-five patients (male: $64 \%$; mean \pm standard deviation age: $65 \pm 13$ years; prevalence of diabetes: $50 \%$ ) were included. The patients' demographic and clinical characteristics, laboratory data, and calcification scores are summarized in Table 1. Both drugs were effective to lowering serum phosphate and urea levels.

After untargeted screening, we selected three carbamylation sites: LVRPEVDVMCTAFHDNEETFLKK, LKCASLQK, and KQTALVELVK (Table 2 and Table S1 of the ESM). These sites are referred to hereafter as LVRP, LKCA, and KQTA respectively. The most frequent carbamylation site was Lys 549 present in the KQTA peptide (Fig. 1). We then developed a multiple reaction monitoring method to reproducibly quantify the proportion of carbamylated albumin (percent carbamylation) on these three-selected peptide sequences (i.e., the ratio between carbamylated and non-carbamylated peptide forms and then by calculating their percentiles) [Table S1 of the ESM]. In this cohort, the most carbamylated site is KQTA, which is $28 \%$ of albumin. At baseline, only the KQTA carbamylation site was

Table 1 Baseline demographic clinical characteristics, laboratory results, and calcification scores in the NICOREN cohort of patients with end-stage kidney disease (ESKD)

\begin{tabular}{ll}
\hline Patients with ESKD $(n=55)$ & \\
Baseline & \\
\hline Demographics & \\
Age (years) & $66.4 \pm 13.1$ \\
Men (\%) & 64 \\
Sevelamer randomization group, $n(\%)$ & $27(49)$ \\
Clinical & \\
BMI (kg/m $\left.{ }^{2}\right)$ & $27.5 \pm 6.1$ \\
History of CVD (\%) & 49 \\
Diabetes mellitus $(\%)$ & 50 \\
Dialysis dose (Kt/Vurea) & $1.28 \pm 0.3$ \\
Pre-dialysis systolic BP (mmHg) & $147 \pm 28$ \\
Laboratory data & \\
Serum urea (mmol/L) & $25.5 \pm 8.1$ \\
Serum albumin (g/dL) & $37.5 \pm 3.5$ \\
Serum protein (g/dL) & $61.8 \pm 5.9$ \\
Peptide LVRP (\%) & $1.5(1.2-2.1)$ \\
Peptide KQTA (\%) & $25.1(21.2-32.7)$ \\
Peptide LKCA (\%) & $4.2(1.9-29.8)$ \\
Calcification scores & $8(3-12)$ \\
Adragao score (vascular calcification) & $3(1-5)$ \\
Kauppila score (aortic calcification) &
\end{tabular}

$A U C$ area under the curve, $B M I$ body mass index, $B P$ blood pressure, $C V D$ cardiovascular disease, LVRP KQTA LKCA three albumin carbamylation sites LVRPEVDVMCTAFHDNEETFLKK, LKCASLQK, and KQTALVELVK

Data are quoted as the mean \pm standard deviation or median (25th75th percentile) for variables with a non-Gaussian distribution and (for qualitative variables) the frequency (percentage)

Percdent carbamylation was calculated as follows: \% carbamylation $=(\mathrm{AUC}$ for the carbamylated peptide/AUC for the non-carbamylated peptide) 
significantly correlated with the urea level in patients with $\operatorname{ESKD}(p=0.042)$ (Fig. 2).

Using multiple reaction monitoring, we demonstrated that the percent carbamylation did not differ when comparing the groups after 24 weeks of treatment with sevelamer or nicotinamide (Table 2). Furthermore, the percent carbamylation values for LVRP and KQTA were strongly correlated, whereas the values for LKCA and KQTA were not (Fig. 3).
The correlation coefficients for the various percent carbamylation values at baseline and the patients' Kauppila and Adragao vascular calcification scores are given in Table 3. None of the correlations was statistically significant.

Table 2 Comparison of carbamylation parameters at baseline and after 24 weeks of treatment in each group

\begin{tabular}{|c|c|c|c|c|c|}
\hline & \multicolumn{2}{|c|}{ Sevelamer group $(n=19)$} & \multicolumn{2}{|c|}{ Nicotinamide group $(n=10)$} & \multirow{2}{*}{$\begin{array}{l}\text { Effect of treat- } \\
\text { ment/time/inter- } \\
\text { action }\end{array}$} \\
\hline & Baseline & 24 weeks & Baseline & 24 weeks & \\
\hline Serum albumin $(\mathrm{g} / \mathrm{L})$ & $37.9 \pm 4.0$ & $36.1 \pm 3.4$ & $37.8 \pm 1.5$ & $37.2 \pm 4.1$ & $0.685 / 0.586 / 0.970$ \\
\hline Serum protein $(\mathrm{g} / \mathrm{L})$ & $62.8 \pm 5.3$ & $60.2 \pm 6.7$ & $60.7 \pm 3.5$ & $57.9 \pm 6.5$ & $0.250 / 0.036 / 0.957$ \\
\hline Serum urea $(\mathrm{mmol} / \mathrm{L})$ & $26.2 \pm 7.0$ & $23.4 \pm 5.4$ & $27.3 \pm 6.4$ & $22.6 \pm 5.8$ & $0.963 / 0.001 / 0.344$ \\
\hline Dialysis dose (Kt/Vurea) & $1.3 \pm 0.2$ & $1.3 \pm 0.3$ & $1.3 \pm 0.3$ & $1.2 \pm 0.7$ & $0.961 / 0.373 / 0.133$ \\
\hline Peptide LVRP (\%) & $1.6(1.3-2.1)$ & $1.5(1.3-1.9)$ & $1.8(1.2-2.1)$ & $1.8(1.5-1.9)$ & $0.766 / 0.675 / 0.617$ \\
\hline Peptide KQTA (\%) & $25.1(23.8-31.3)$ & $26.5(22.5-30.7)$ & $27.4(19.7-32.9)$ & $30.7(25.5-33.5)$ & $0.897 / 0.098 / 0.063$ \\
\hline Peptide LKCA (\%) & $10.8(1.7-37.9)$ & $3.4(2.0-31.9)$ & $4.1(1.2-14.3)$ & $2.7(1.1-12.5)$ & $0.275 / 0.399 / 0.945$ \\
\hline
\end{tabular}

$A U C$ area under the curve, LVRP KQTA LKCA three albumin carbamylation sites LVRPEVDVMCTAFHDNEETFLKK, LKCASLQK, and KQTALVELVK

Percent carbamylation was calculated as follows: \% carbamylation $=($ AUC for the carbamylated peptide/AUC for the non-carbamylated peptide
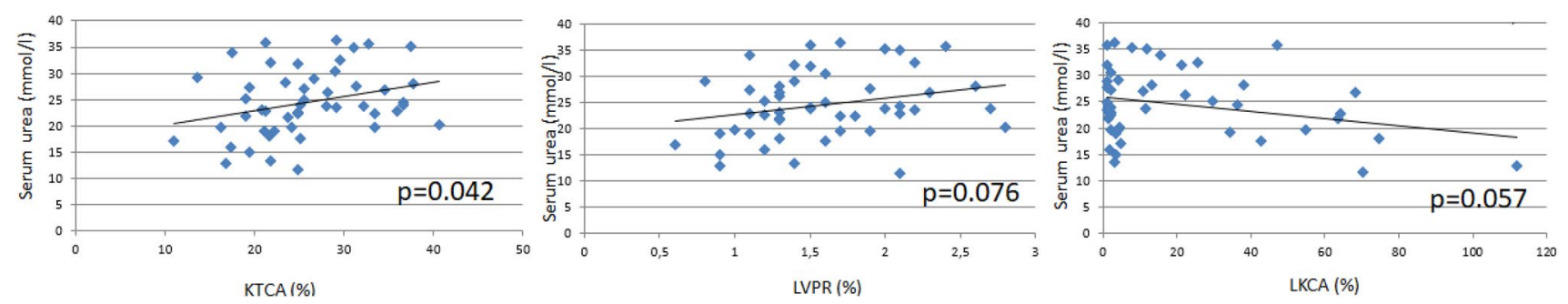

Fig. 2 Correlation between serum urea and three albumin carbamylation sites
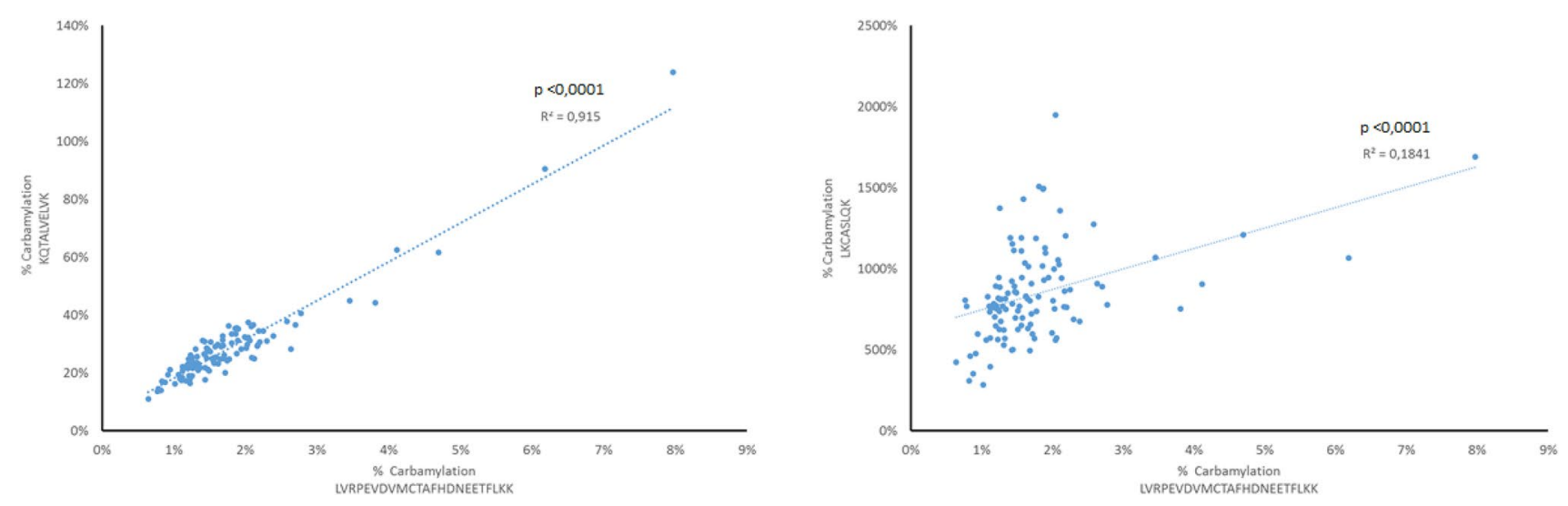

Fig. 3 Correlations between pairs of selected peptides. Percent carbamylation was calculated as follows: $\%$ carbamylation $=($ area under the curve for the carbamylated peptide/area under the curve for the non-carbamylated peptide) 
Table 3 Spearman coefficients (R) for the correlation between serum albumin carbamylation and vascular calcification scores in 47 patients with end-stage kidney disease

\begin{tabular}{lrc}
\hline Variables & $R$ value & $p$ value \\
\hline Kauppila score & & \\
Peptide LVRP & 0.192 & 0.338 \\
Peptide KQTA & 0.054 & 0.789 \\
Peptide LKCA & -0.141 & 0.482 \\
Adragao score & & \\
Peptide LVRP & -0.058 & 0.758 \\
Peptide KQTA & -0.211 & 0.255 \\
Peptide LKCA & 0.033 & 0.858 \\
\hline
\end{tabular}

LVRP KQTA LKCA three albumin carbamylation sites LVRPEVDVMCTAFHDNEETFLKK, LKCASLQK, and KQTALVELVK

\section{Discussion}

In the present study, we developed a method for quantifying albumin carbamylation and then used it to quantify serum albumin carbamylation in patients included in the NICOREN trial. After 24 weeks of treatment in patients with ESKD, we observed a high level of carbamylated albumin at baseline in three sites on the protein but the level of albumin carbamylation did not decrease in either of the two groups, although both drugs were effective at lowering serum phosphate and urea levels. Moreover, we did not find a significant association between carbamylated albumin and the presence of vascular calcification.

Our analytical method was based in trypsin digestion, which we think is more suitable than Glu-c digestion used by others [17]. Glu-c digestion is known to be sensitive to the experimental conditions and is not very reproducible. In preliminary experiments, we were unable to obtain reproducible results with Glu-c. Furthermore, our trypsin digestion procedure took only 3 hours (compared with 24 hours for Glu-c) and was reproducible. Despite these differences in methods, the peptides LVRP and KQTA that we identified and quantified for albumin carbamylation were also identified by Berg et al. Moreover, the percent carbamylation observed in the present study was similar to that reported by Berg et al. [17]. Two of our three peptides were perfectly correlated, and thus one of them could be used to express the percent carbamylation for albumin. LKCA was not an optimal marker because its percent carbamylation was not correlated with those of LVRP and KQTA. Hence, LKCA might have been carbamylated via a different pathway.

Based on KQTA and LVRP in patients with ESKD, we found that 28.14 and $1.78 \%$ of serum albumin molecules were carbamylated, respectively. In a study of non-uremic patients, Berg et al. found a mean value of $0.4 \%$ [15]. In fact, percent carbamylation for albumin was correlated with timeaveraged blood urea concentrations and was twice as high in patients with ESKD than in non-uremic subjects [18]. It is noteworthy that the serum amino acid concentration is related to the time since the initiation of hemodialysis, inflammation, and malnutrition. Indeed, Małgorzewicz et al. demonstrated that patients with ESKD have abnormal amino acid profiles [19]. Our results emphasize the extent of carbamylation in patients with ESKD.

Neither sevelamer nor nicotinamide appeared to have impacted albumin carbamylation at different sites (LVRP, LKCA, and KQTA) after 24 weeks of treatment, despite a decrease in the serum urea concentration in the sevelamer and nicotinamide groups. In contrast, Di Iorio et al. reported that nutritional treatments (a Mediterranean diet and a very low protein diet) were associated with a significant decrease in serum levels of urea and in protein carbamylation in patients with chronic kidney disease (CKD) grades 3B-4 [20]. Vanholder et al. reported that urea is a major source of the carbamylated compounds that trigger profibrotic damage in mesangial cells, concomitant structural changes in collagen, vascular damage, and endothelial cell apoptosis, thus promoting atherosclerosis [21]. Moreover, the results of Shaykh et al.'s in vitro study suggested that carbamylation affects the metabolism of mesangial cells and promotes glomerular sclerosis through a specific effect in matrix proteins such as collagens I and IV [22]. There appears to be a weak correlation between protein carbamylation (as evaluated by plasma levels of protein-bound homocitrulline) and urea levels [6]. However, other mechanisms beyond urea, such as a possible role of myeloperoxidase, that cause protein carbamylation in patients with ESKD may also exist but remain to be characterized.

Carbamylation molecules are acknowledged prognostic biomarkers in CKD. Indeed, protein carbamylation is positively associated with all-cause mortality in patients with CKD, regardless of the stage $[6,18,23]$. This elevated mortality might be due to the impact of carbamylation on vascular calcification, which is a solid predictor of mortality in patients with CKD. Recently, Mori et al.'s studies including ex vivo and in vivo mice models demonstrated that protein carbamylation exacerbates vascular calcification by impairing mitochondrial function, which in turn results in the downregulation of ENPP1 expression [4]. ENPP1 produces pyrophosphate, which is a potent inhibitor of vascular calcification. The importance of ENPP1 in the pathogenesis of vascular calcification has been confirmed in mice and in humans [23, 24]. However, our data did not reveal an association between albumin carbamylation and vascular calcification in patients with ESKD. Further studies with larger sample sizes and longer follow-up periods are now needed to explore the potential involvement of the carbamylation of other molecules in vascular calcification.

Our study had several limitations, including a relatively small sample size, a treatment period of only 24 
weeks (which may have been too short to assess long-term changes), and a drop-out rate of $45 \%$ in the nicotinamide group (compared with $10 \%$ in the sevelamer group). Our method for evaluating calcification (on plain radiographs) is semi-quantitative and strongly observer dependent. Moreover, the present study did not feature a control group of individuals not treated with sevelamer or nicotinamide. A comparison with a control group might provide additional information about the effect of phosphate binders on albumin carbamylation. However, it should be noted that these phosphate binders are widely taken to control the elevated serum phosphate levels in patients with ESKD; hence, obtaining a large non-treated control group would be difficult. Last, the use of isotope measurements would have strengthened the study.

\section{Conclusions}

The present study confirms that serum albumin carbamylation is elevated in patients with ESKD and shows its effect beyond the LVRP site. However, we did not find any evidence of an impact of treatment with sevelamer or nicotinamide on serum albumin carbamylation. Therapeutic strategies to lower carbamylation load should probably be focused on direct anti-carbamylation processes and/or potentially anti-inflammatory therapies.

Supplementary Information The online version contains supplementary material available at https://doi.org/10.1007/s40268-021-00350-7.

Acknowledgements We thank Prof. Albert Fournier, who initiated this clinical study. We also thank Amiens University Hospital, and especially the Clinical Research and Innovation Directorate (for logistical support), the Clinical Research Center (for study management), and the Department of Nephrology Internal Medicine, Dialysis, Transplantation and Intensive Care. Last, we thank the patients and physicians at the 18 dialysis centers for their participation in the NICOREN trial.

\section{Declarations}

Funding The study was funded by a grant from the French Government's Inter-Regional Hospital Research Program (PHRC IR08: 2008004673-17).

Conflict of interest Ziad A. Massy reports grants and Congress Travel support from Amgen, Baxter, Otsuka, and Sanofi-Genzyme, grants from the French Government, MSD, GSK, Lilly, and FMC, and Receipt of honoraria or consultation fees for the Charities from Daichi and Astellas, outside the submitted work.

Ethics approval The study protocol was approved by a local investigational review board (CPP Nord Ouest II, Amiens, France; reference: 2008-004673-17) and implemented in accordance with the ethical principles of the Declaration of Helsinki (ClinicalTrials.gov registration number: NCT01011699).
Consent to participate All participants gave their written agreement to participate in the study.

Consent for publication All participants gave their written agreement to publication of the study data.

Availability of data and material Not applicable.

Code availability Not applicable.

Author contributions Each author contributed intellectual content. ZM and SLB conceived the research idea; AL drafted the manuscript, performed the statistical analysis, and analyzed the data; MAR, JLS, and SLB performed the albumin carbamylation assays; all authors performed a critical analysis of the manuscript, ZM supervised the study.

Open Access This article is licensed under a Creative Commons Attribution-NonCommercial 4.0 International License, which permits any non-commercial use, sharing, adaptation, distribution and reproduction in any medium or format, as long as you give appropriate credit to the original author(s) and the source, provide a link to the Creative Commons licence, and indicate if changes were made. The images or other third party material in this article are included in the article's Creative Commons licence, unless indicated otherwise in a credit line to the material. If material is not included in the article's Creative Commons licence and your intended use is not permitted by statutory regulation or exceeds the permitted use, you will need to obtain permission directly from the copyright holder. To view a copy of this licence, visit http://creativecommons.org/licenses/by-nc/4.0/.

\section{References}

1. Verbrugge FH, Tang WHW, Hazen SL. Protein carbamylation and cardiovascular disease. Kidney Int. 2015;88:474-8.

2. Delanghe S, Delanghe JR, Speeckaert R, Van Biesen W, Speeckaert MM. Mechanisms and consequences of carbamoylation. Nat Rev Nephrol. 2017;13:580-93.

3. Long J, Vela Parada X, Kalim S. Protein carbamylation in chronic kidney disease and dialysis. Adv Clin Chem. 2018;87:37-67.

4. Mori D, Matsui I, Shimomura A, Hashimoto N, Matsumoto A, Shimada K, et al. Protein carbamylation exacerbates vascular calcification. Kidney Int. 2018;94:72-90.

5. Drechsler C, Kalim S, Wenger JB, Suntharalingam P, Hod T, Thadhani RI, et al. Protein carbamylation is associated with heart failure and mortality in diabetic patients with end-stage renal disease. Kidney Int. 2015;87:1201-8.

6. Koeth RA, Kalantar-Zadeh K, Wang Z, Fu X, Tang WHW, Hazen SL. Protein carbamylation predicts mortality in ESRD. J Am Soc Nephrol. 2013;24:853-61.

7. Lenglet A, Liabeuf S, El Esper N, Brisset S, Mansour J, LemaireHurtel A-S, et al. Efficacy and safety of nicotinamide in haemodialysis patients: the NICOREN study. Nephrol Dial Transplant. 2017;32:870-9.

8. Lenglet A, Fabresse N, Taupin M, Gomila C, Liabeuf S, Kamel $S$, et al. Does the administration of sevelamer or nicotinamide modify uremic toxins or endotoxemia in chronic hemodialysis patients? Drugs. 2019;79:855-62.

9. Nikolov IG, Joki N, Maizel J, Lacour B, Drüeke TB, Massy ZA. Pleiotropic effects of the non-calcium phosphate binder sevelamer. Kidney Int. 2006;70:S16-23. 
10. Lenglet A, Liabeuf S, Guffroy P, Fournier A, Brazier M, Massy ZA. Use of nicotinamide to treat hyperphosphatemia in dialysis patients. Drugs RD. 2013;13:165-73.

11. Moberg L, Olsson A, Berne C, Felldin M, Foss A, Källen R, et al. Nicotinamide inhibits tissue factor expression in isolated human pancreatic islets: implications for clinical islet transplantation. Transplantation. 2003;76:1285-8.

12. Hiromatsu Y, Sato M, Yamada K, Nonaka K. Inhibitory effects of nicotinamide on recombinant human interferon-gamma-induced intercellular adhesion molecule-1 (ICAM-1) and HLA-DR antigen expression on cultured human endothelial cells. Immunol Lett. 1992;31:35-9.

13. Biedroń R, Ciszek M, Tokarczyk M, Bobek M, Kurnyta M, Słominska EM, et al. 1-Methylnicotinamide and nicotinamide: two related anti-inflammatory agents that differentially affect the functions of activated macrophages. Arch Immunol Ther Exp (Warsz). 2008;56:127-34.

14. Lappas M, Permezel M. The anti-inflammatory and antioxidative effects of nicotinamide, a vitamin $\mathrm{B}(3)$ derivative, are elicited by FoxO3 in human gestational tissues: implications for preterm birth. J Nutr Biochem. 2011;22:1195-201.

15. Berg AH, Drechsler C, Wenger J, Buccafusca R, Hod T, Kalim S, et al. Carbamylation of serum albumin as a risk factor for mortality in patients with kidney failure. Sci Transl Med. 2013;5:175ra29.

16. Kauppila LI, Polak JF, Cupples LA, Hannan MT, Kiel DP, Wilson PW. New indices to classify location, severity and progression of calcific lesions in the abdominal aorta: a 25-year follow-up study. Atherosclerosis. 1997;132:245-50.

17. Adragao T, Pires A, Lucas C, Birne R, Magalhaes L, Gonçalves $\mathrm{M}$, et al. A simple vascular calcification score predicts cardiovascular risk in haemodialysis patients. Nephrol Dial Transplant. 2004;19:1480-8.

18. Wang Z, Nicholls SJ, Rodriguez ER, Kummu O, Hörkkö S, Barnard J, et al. Protein carbamylation links inflammation, smoking, uremia and atherogenesis. Nat Med. 2007;13:1176-84.

19. Małgorzewicz S, Debska-Slizień A, Rutkowski B, LysiakSzydłowska W. Serum concentration of amino acids versus nutritional status in hemodialysis patients. J Ren Nutr. 2008;18:239-47.

20. Di Iorio BR, Marzocco S, Bellasi A, De Simone E, Dal Piaz F, Rocchetti MT, et al. Nutritional therapy reduces protein carbamylation through urea lowering in chronic kidney disease. Nephrol Dial Transplant. 2018;33:804-13.

21. Vanholder R, Gryp T, Glorieux G. Urea and chronic kidney disease: the comeback of the century? (in uraemia research). Nephrol Dial Transplant. 2018;33:4-12.

22. Shaykh M, Pegoraro AA, Mo W, Arruda JA, Dunea G, Singh AK. Carbamylated proteins activate glomerular mesangial cells and stimulate collagen deposition. J Lab Clin Med. 1999;133:302-8.

23. Speer T, Owala FO, Holy EW, Zewinger S, Frenzel FL, Stähli BE, et al. Carbamylated low-density lipoprotein induces endothelial dysfunction. Eur Heart J. 2014;35:3021-32.

24. Harmey D, Hessle L, Narisawa S, Johnson KA, Terkeltaub R, Millán JL. Concerted regulation of inorganic pyrophosphate and osteopontin by akp2, enpp1, and ank: an integrated model of the pathogenesis of mineralization disorders. Am J Pathol. 2004;164:1199-209.

25. Rutsch F, Ruf N, Vaingankar S, Toliat MR, Suk A, Höhne W, et al. Mutations in ENPP1 are associated with "idiopathic" infantile arterial calcification. Nat Genet. 2003;34:379-81.

\section{Authors and Affiliations}

\section{Aurelie Lenglet ${ }^{1,2} \cdot$ Mohamad Ali Rahali $^{3} \cdot$ François-Ludovic Sauvage $^{3} \cdot$ Sophie Liabeuf ${ }^{1,4} \cdot$ Gabriel Choukroun $^{1,5}$. Marie Essig ${ }^{6,7} \cdot$ Souleiman El Balkhi ${ }^{3,8} \cdot$ Ziad A. Massy $^{6,7}$}

1 EA7517, MP3CV Laboratory, CURS, Faculty of Pharmacy, Jules Verne University of Picardie, Amiens, France

2 Pharmacy, Amiens University Hospital, Amiens, France

3 INSERM, IPPRITT, U1248, Limoges, France

4 Division of Pharmacology, Amiens University Hospital, Amiens, France

5 Department of Nephrology Dialysis and Transplantation, Amiens University Hospital, Amiens, France
6 INSERM U-1018, Centre de Recherche en Épidémiologie et Santé des Populations (CESP), Paris-Saclay University (PSU), University of Paris Ouest-Versailles-Saint-Quentinen-Yvelines (UVSQ), Equipe 5, Villejuif, Paris, France

7 Division of Nephrology, Ambroise Paré Hospital, Paris Ile de France Ouest University, 9 Avenue Charles de Gaulle, 92104 Boulogne Billancourt Cedex, France

8 Department of Pharmacology, Toxicology and Pharmacovigilance, CHU Limoges, Limoges, France 